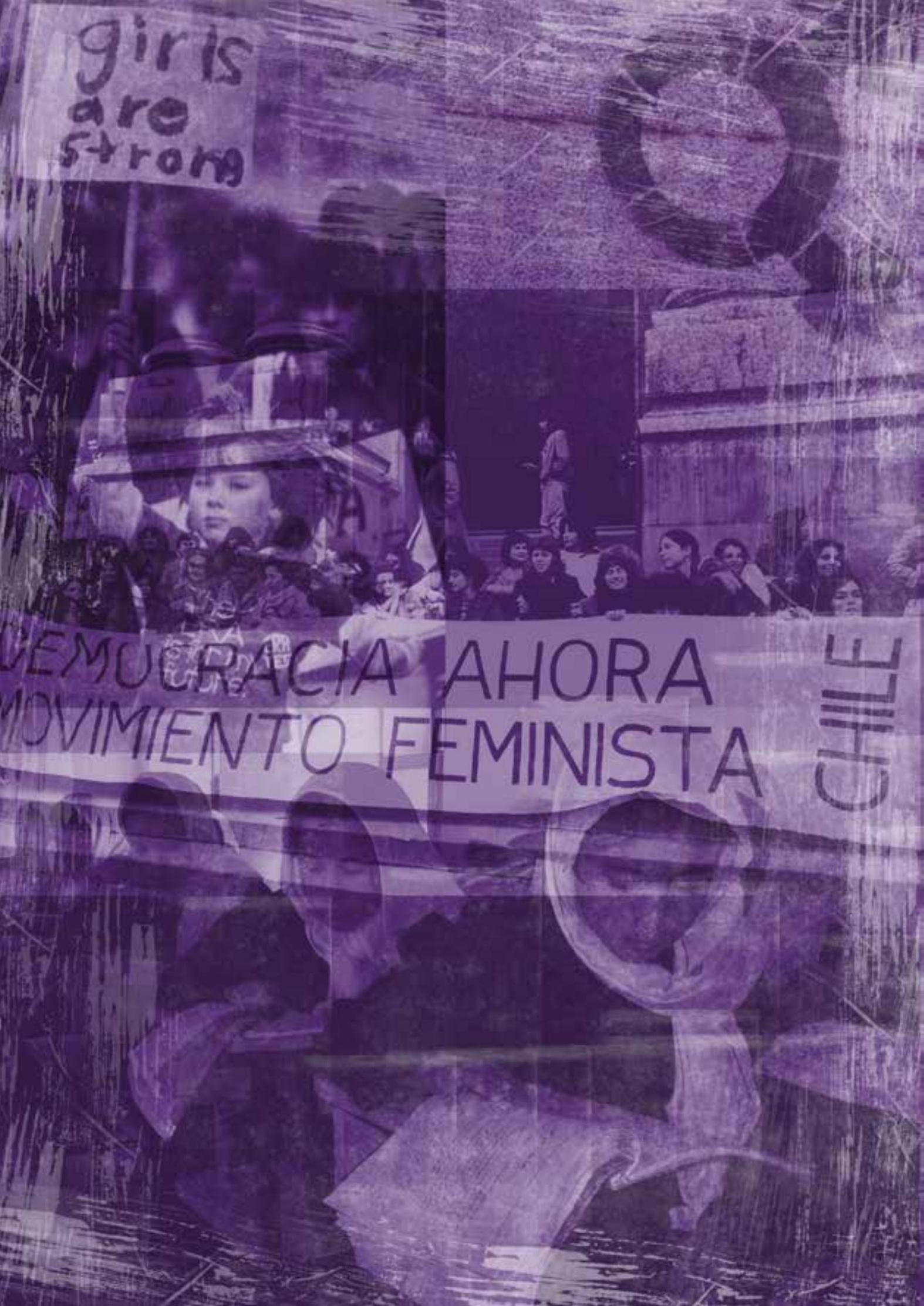




\title{
Presentación: \\ Herta Herzog como antídoto
}

DoI: $10.15213 /$ redes.n11.p18

\author{
AMPARO HUERTAS BAILÉN
}

Ante el volumen de contenido sexista que todavía circula en los medios, en muchas ocasiones es fácil olvidarse de los pasos ya dados en la dirección correcta, hacia la igualdad. Sí, se ha avanzado, pero, debido a que estos temas requieren de un compromiso político y ético por parte de los equipos de investigación, cuesta aceptar la evidencia de que hay que seguir trabajando o, quizá sería mejor decir, de que hay que seguir esforzándose. Ante una sociedad hipersexualizada sostenida por una potente industria cultural (RINGROSE ET AL., 2013; ADRIAENS Y VAN BAUWEL, 2011), es francamente difícil hacer valer un discurso público asentado en la perspectiva de género. $\mathrm{Si}$, además, añadimos la falta de recursos financieros destinados a I+D, el panorama es todavía más desalentador. Pero no quisiéramos contribuir aquí a un espíritu pesimista, todo lo contrario. De hecho, para esos momentos de duda sobre el camino ya recorrido, les queríamos ofrecer en esta presentación, entre otras cosas, un antídoto.

Una de las primeras investigaciones que hay registradas sobre el consumo mediático de las mujeres es un trabajo de Herta Herzog ${ }^{1}$ acerca de las oyentes de radio, publicado en 1944. El proyecto de Herzog trataba de responder a dos preguntas: ¿cuáles son las características sociales de las mujeres que escuchan los seriales radiofónicos? y ¿con qué finalidad o utilidad realizan ese consumo? Un planteamiento claro e, incluso, resulta gratificante leer la segunda pregunta porque, de alguna manera, muestra que la autora ya intuía la consideración de un sujeto activo en el proceso de recepción.

El estudio se enmarcaba en el ámbito de los efectos de los medios de comunicación, tema dominante en aquel contexto bélico (Guerras Mundiales) donde gran parte de los trabajos se dedicaban a buscar la clave para una

1 Herta Herzog nació en Viena (1910-2010). Trabajó en la Office of Radio Research, creada en 1937 con fondos de la Rockefeller Foundation. Paul Lazarsfeld fue su primer director. Desde 1940 forma parte de Columbia University (KATz, 1987). En la actualidad, se denomina Institute for Social and Economic Research and Policy (ISERP)

REDES.COM N ${ }^{\circ} 10 \mid 19$ 
eficiente comunicación persuasiva. En los años 70 y 80 del siglo pasado, el estudio de Herzog solía mencionarse en los recopilatorios y textos sobre la evolución de las Teorías de la Comunicación e, incluso, en muchas ocasiones se le calificaba como gran contribución a la formación de la teoría de los usos y gratificaciones.

Pero vayamos ya a lo que queríamos destacar. Estas eran sus hipótesis:

a) Las mujeres viven separadas de su comunidad, con un notable grado de soledad, debido al elevado número de horas que emplean escuchando la radio;

b) las mujeres presentan muy poco interés por las cuestiones intelectuales;

c) las mujeres presentan todavía menor interés por los asuntos públicos;

d) las mujeres, que se caracterizan por sufrir ataques de ansiedad y múltiples frustraciones, encuentran en la radio un medio para compensar ese malestar; $y$, por último,

e) las mujeres tienden a escuchar la radio más que el resto de la población.

Estas afirmaciones ofrecen una clara visión de los proyectos de vida que en aquella época, coincidiendo con el inicio del desarrollo de los estudios de comunicación, se asignaban a las mujeres por el hecho de serlo y de cómo la investigación lo asumía como contexto socio-cultural dado sin ninguna mirada crítica. El dedicar demasiadas horas a la radio se entendía que podía desembocar en el descuido en el cuidado de la familia/comunidad. Los contenidos que les debían interesar eran las historias basadas en el amor romántico y en la mujer ideal (madre y esposa), por encima de las cuestiones públicas, y, como muy bien resumieron Ien Ang y Joke Hermes (1991), los medios de aquellos años daban una imagen de las mujeres irreal y exclusivamente basada en valores conservadores. Por no hablar de la consideración de una innata predisposición enfermiza hacia la histeria, idea que tan adherida se ha mantenido al estereotipo femenino, o el no plantear que esa mayor audición podía deberse a una reclusión en el espacio doméstico.

Para comprobar sus hipótesis, la autora comparó el comportamiento de oyentes con el de no oyentes. $\mathrm{Y}$, como seguramente se habrá despertado la curiosidad por conocer los resultados, aquí están. Herzog tan solo encontró esta diferencia: entre las oyentes de radio se daba una mayor preferencia por las novelas de misterio y por las revistas que trataban temas similares a los de 
los seriales radiofónicos. La autora lo interpretó apuntando que quizá escuchar la radio desencadenaba una mayor habilidad para experimentar sensaciones vicarias (la radio como sustituta de las experiencias directas reales). Un apunte que, aunque podía relacionarse con un modo/deseo de escapar del entorno doméstico, en aquella época, en cambio, sirvió para reforzar el estereotipo de unas mentes fantasiosas. De hecho, en un trabajo previo a este, Herzog (1941) ya había abordado, y más extensamente, las vivencias emocionales y el desarrollo de la fantasía ligado a la audición de seriales radiofónicos por parte de las mujeres.

Esperamos que recordar este tipo de trabajos sirva a lectores y lectoras para recuperar esa agradable sensación de un camino ya recorrido. Pero, evidentemente, la preocupación persiste.

El manual escrito por John L. Sullivan y editado en 2013 por SAGE Publications Inc., con el título Media audiences. Effects, users, institutions, and power, dedica cuatro párrafos al trabajo de Herta Herzog. La información aparece acompañada de una fotografía en la que se ve a una mujer anónima. Esta se describe diciendo "Joven mujer enciende la radio a principios de los años 30 " y, a continuación, se indica la procedencia de la imagen. Para concluir la referencia, el libro tan solo apunta que el trabajo de Herzog inspiró siguientes estudios sobre las experiencias de las audiencias. Nada sobre la cuestión de género, ningún comentario con perspectiva crítica. Es decir, todavía - déjenme ser optimista y emplear así este adverbio - no se ha conseguido que la sensibilidad sobre las cuestiones de género llegue a los manuales que hoy están utilizando estudiantes universitarios/as.

Y, si miramos al entorno profesional, el panorama tampoco es muy alentador. En noviembre de 2014, tuvimos la oportunidad de participar en la $I$ Jornada sobre mujeres $y$ radio ${ }^{2}$. El encuentro puso en evidencia la permanencia de la desigualdad de género desde múltiples ángulos: el acceso a cargos directivos, las diferencias salariales, las relaciones laborales entre compañeros y compañeras, el escaso poder que tienen las mujeres en algunas redacciones a la hora de evitar la emisión de noticias con rasgos sexistas o la dificultad de cuestionar comentarios claramente machistas cuando comparten micrófono en un programa. Pero quizá lo que más nos llamó la atención fue percibir las contradicciones en las intervenciones de las locutoras más jóvenes. De alguna

2 Fue una iniciativa del Departamento de Filología y Comunicación de la Universitat de Girona (UdG), de l'Institut Català de les Dones (ICD) y del Col•legi de Periodistes de Catalunya. En su organización, también colaboró l'Observatori de la Ràdio de Catalunya y el Instituto de la Comunicación-Universitat Autònoma de Barcelona (InCom-UAB).

REDES.COM N $\mathrm{N}^{\mathrm{O}} 11 \mid 21$ 
manera, les costaba ver y aceptar la situación, incluso cuando ellas mismas habían vivido decisiones discriminatorias.

No cabe duda de que sigue siendo prioritario abordar, analizar y dar visibilidad a esta problemática. Tanto para avanzar en su conocimiento como para contribuir a la definición de estrategias que permitan acabar con estas desigualdades en todos los terrenos, mediático, laboral, académico, centros escolares, familia,...

En el ámbito de las teorías feministas existen perspectivas que afirman haberse superado la Segunda Ola, caracterizada por la defensa de los derechos y el reconocimiento de las mujeres, y se señala estar ya viviendo la Tercera, en la que la lucha se centra en el reconocimiento definitivo de la diversidad. Si atendemos al entorno comunicativo (socio-tecnológico) que nos rodea, resulta muy difícil aceptar esta visión de la historia formada por ciclos contiguos que implican la acumulación automática de conocimientos y logros sociales. Nuestro entorno parece más bien revelar múltiples contradicciones acompañadas de retrocesos.

Los textos recogidos aquí ponen sobre la mesa, una vez más, la existencia de un consolidado pensamiento crítico frente a la sociedad heteropatriarcal y frente a los estereotipos de género que de ella surgen. Algunos de ellos se adentran a analizar la actividad digital, considerándola esta como una actividad social (y real) más y sin caer en el deslumbramiento que a veces produce lo tecnológico. Los textos proceden de equipos de investigación ubicados en diferentes países (Argentina, España, Portugal, Brasil), por lo que se ofrece una excelente panorámica sobre las tendencias de estudio en materia de género.

Sobre la construcción mediática del género, se recogen dos aportaciones. El texto de Hadriel Geovani da Silva Theodoro aborda la participación de Cristina Wurst en el festival de Eurovision. El autor supo aprovechar el momento para analizar la reacción de los medios ante esa imagen transgresora (performance), que aúna características masculinas y femeninas, a partir de uno de los eventos televisivos con mayor resonancia global existentes. Soraya Barreto analiza el discurso publicitario que las revistas portuguesas dirigidas a los hombres elaboran en torno a la masculinidad, partiendo de su notable rol como difusoras de modelos de estilos de vida. Aunque los sectores de la cosmética y de la moda muestran un elevado interés comercial, al igual que sucede en la prensa destinada a las mujeres, la investigadora también señala aspectos innovadores.

Con la mirada puesta en la noción de ciudadanía comunicativa, Gabriela Bard Wigdor expone cómo la situación de desigualdad de género en el acceso a derechos y al espacio público implica no sólo la exclusión de las mujeres y 
otros sectores no dominantes sino también la legitimación y perpetuación de ciertas voces y necesidades, vinculadas a los varones y a los grupos dominantes. A partir del análisis de las prácticas de participación de las mujeres en dos barrios populares de Córdoba (Argentina), insiste en la idea de que participar en democracia no es únicamente votar, también se ha de poder participar en las políticas que conciernen a la distribución de la riqueza tanto material, como cultural y simbólica.

Con una perspectiva feminista, Júlia Araújo Mendes analiza la trayectoria de "Enredadas Mujeres", programa emitido por una radio libre de la ciudad de Valencia (España). Araújo, aunque se cuestiona la pertinencia de un producto que ya sabe de antemano que no tendrá una audiencia masiva, acaba argumentando la necesidad de los mismos con el fin de luchar contra la sociedad heteropatriarcal. Y, desde la ciudad de Santa Maria, en Rio Grande do Sul-Brasil, nos llega un análisis del movimiento Marcha das Vadias (Marcha de las Putas) y, más concretamente, de cómo este emplea Facebook. El trabajo, de Tainan Pauli Tomazetti y Liliane Dutra Brignol, constata el importante papel que desempeña esta red social digital, no solo en lo que se refiere a la visibilidad sino también a la organización de este movimiento social.

Sobre el entretenimiento digital, este monográfico incluye un trabajo acerca de los videojuegos. Héctor Puente Bienvenido y Amparo Lasén Díaz entienden las partidas online como una especie de coreografía colectiva, donde se construye género y prácticas desiguales. Con su estudio, centrado en el célebre videojuego de rol de acción multijugador (aRPG) Diablo III, ambos autores acaban demostrando un elevado grado de sexismo en el desarrollo de las partidas.

En definitiva, no nos cabe la menor duda de que este monográfico supone un paso más en esa buena dirección que apuntábamos al inicio, por pequeño que este sea. Y, para cuando lo necesiten, recuerden que aquí siempre encontrarán un antídoto. 


\section{REFERENCIAS}

ADRIANES, F. y VAN BAUWEL, S. (2011). "Remove from marked Records Sex and the City: a postfeminist point of view? Or how popular culture functions as a channel for feminist discourse. Journal of Popular Culture (Boston), vol. 47, No. 1, pp. 174-195.

ANG, I. y HERMES, J. (1991). Gender and/in Media consumption. En CURRAN, J. y GUREVITCH, M. (EDs.) Mass Media and Society. Londres: Edward Arnold, pp. 307-328.

HERZOG, H. (1941). On borrowed experience. En Studies in Philosophy and Social Science, 9, 65-99.

HERZOG, H. (1944). What do really know about daytime serial listeners. En Lazarsfeld, P.F. y Stanton, F.N. (EDS.) Radio Research 1942-1943. Nueva York: Duell, Sloan \& Pearce, pp. 3-33.

KATZ, E. (1987). Communications Research Since Lazarsfeld. Public Opinion Quarterly, 51 (4 Part 2), s25-S45. Disponible en: [http://repository.upenn. edu/cgi/viewcontent.cgi?article $=1254 \&$ context $=$ asc_papers]

RINGROSE, J.; GILL, R.; LIVINGSTONE, S.; HARVE, L. (2013). Teen girls, sexual double standards and 'sexting': Gendered value in digital image exchange, Feminist Theory, vol. 14, No. 3, pp. 305-323.

SULLIVAN, J.L. (2013) Media audiences. Effects, Users, Institutions, and Power. Los Angeles: sage Publications Inc. 